\title{
The Caenorhabditis elegans NF2/Merlin Molecule NFM-1 Nonautonomously Regulates Neuroblast Migration and Interacts Genetically with the Guidance Cue SLT-1/Slit
}

\author{
Matthew P. Josephson, Rana Aliani, Megan L. Norris, Matthew E. Ochs, Mahekta Gujar, \\ and Erik A. Lundquist ${ }^{1}$ \\ Department of Molecular Biosciences, Program in Molecular, Cellular, and Developmental Biology, University of Kansas, Lawrence, \\ Kansas 66046 \\ ORCID ID: 0000-0001-6819-4815 (E.A.L.)
}

\begin{abstract}
During nervous system development, neurons and their progenitors migrate to their final destinations. In Caenorhabditis elegans, the bilateral Q neuroblasts and their descendants migrate long distances in opposite directions, despite being born in the same posterior region. QR on the right migrates anteriorly and generates the AQR neuron positioned near the head, and QL on the left migrates posteriorly, giving rise to the PQR neuron positioned near the tail. In a screen for genes required for AQR and PQR migration, we identified an allele of $n f m-1$, which encodes a molecule similar to vertebrate NF2/Merlin, an important tumor suppressor in humans. Mutations in NF2 lead to neurofibromatosis type II, characterized by benign tumors of glial tissues. Here we demonstrate that in C. elegans, nfm-1 is required for the ability of Q cells and their descendants to extend protrusions and to migrate, but is not required for direction of migration. Using a combination of mosaic analysis and cell-specific expression, we show that NFM-1 is required nonautonomously, possibly in muscles, to promote Q lineage migrations. We also show a genetic interaction between $n f m-1$ and the C. elegans Slit homolog s/t-1, which encodes a conserved secreted guidance cue. Our results suggest that NFM-1 might be involved in the generation of an extracellular cue that promotes Q neuroblast protrusion and migration that acts with or in parallel to SLT-1. In vertebrates, NF2 and Slit2 interact in axon pathfinding, suggesting a conserved interaction of NF2 and Slit2 in regulating migratory events.
\end{abstract}

KEYWORDS Neuronal migration; Merlin; NFM-1; Q cells; C. elegans

A critical process in nervous system development is the directed migration of neurons to precise destinations. Directed migration is a complex process that requires integration of extracellular cues into cytoskeletal changes, which guide the cell to a specific location. In Cenorhabditis elegans, the $Q$ neuroblasts are an established system to study directed cell migrations (Middelkoop and Korswagen 2014). The Q neuroblasts on the right (QR) and left (QL) are born in the posterior region of the worm yet migrate in opposite directions (Sulston and Horvitz 1977; Salser and Kenyon 1992; Salser et al. 1993). Each undergoes an identical pattern of cell division and cell

Copyright (C) 2017 by the Genetics Society of America

doi: 10.1534/genetics.116.191957

Manuscript received May 23, 2016; accepted for publication November 23, 2016 ; published Early Online December 2, 2016.

${ }^{1}$ Corresponding author: Department of Molecular Biosciences, Program in Molecular, Cellular, and Developmental Biology, University of Kansas, 1200 Sunnyside Ave.,

5049 Haworth Hall, Lawrence, KS 66046. E-mail: erikl@ku.edu death to produce three neurons each: SDQL, PVM, and PQR from QL, and SDQR, AVM, and AQR from QR (Figure 1).

$\mathrm{QL}$ is born on the left side of the animal and migrates posteriorly over the seam cell V5 before dividing (Honigberg and Kenyon 2000; Chapman et al. 2008). During this initial migration, QL detects a posteriorly derived EGL-20/Wnt signal, which through canonical Wnt signaling initiates transcription of mab-5/Hox (Salser and Kenyon 1992). MAB-5 drives further posterior migration of the QL lineage, resulting in the QL.a descendant PQR migrating to the tail near the anus and posterior phasmid ganglion.

QR is born on the right side of the animal and migrates anteriorly over the seam cell V4 and away from the EGL-20/ Wnt signal (Salser et al. 1993; Harris et al. 1996; Salser and Kenyon 1996). QR does not initiate mab-5 expression in response to Wnt and continues to migrate anteriorly. After division, QR.a undergoes an identical pattern of cell divisions 
A

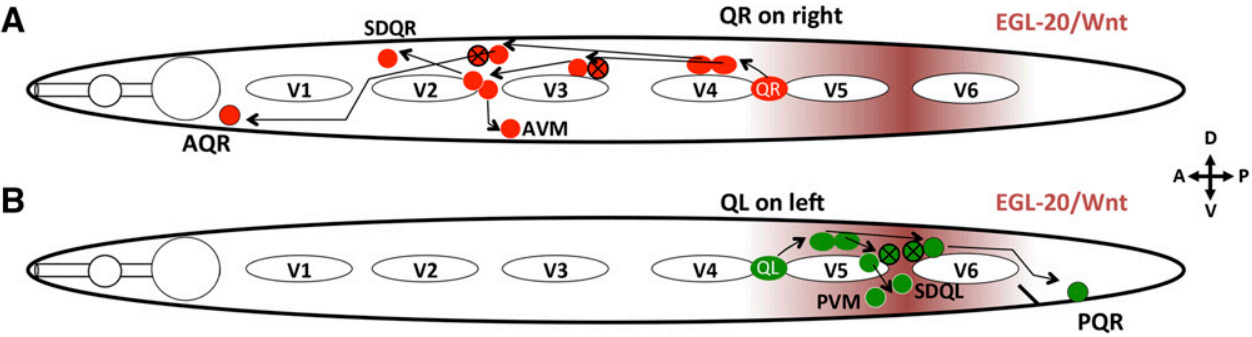

C

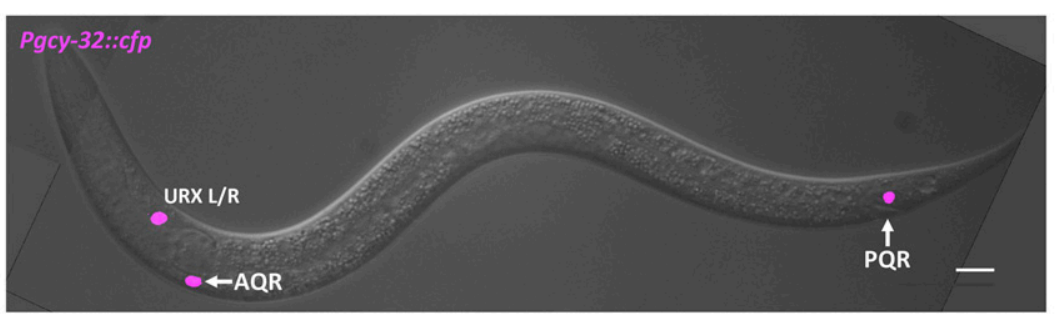

Figure 1 Migration of $Q R$ and $Q L$ descendants. (A and B) Diagrams representing the migration and cell division pattern of QR on the right side (A) and QL on the left side (B) in the L1 animal, showing birthplace of the $\mathrm{Q}$ neuroblasts and approximate locations of the Q descendants. Maroon shading represents the posteriorly derived EGL-20/Wnt signal. White ovals are hypodermal seam cells V1-V6. Circles with black $X$ indicate cells that undergo programmed cell death after cell division. Dorsal is up, anterior to the left. (C) Merged DIC and fluorescent micrograph showing location of $\mathrm{Q}$ descendants $\mathrm{AQR}$ and $\mathrm{PQR}$ in an adult wild-type animal. Pgcy-32:: cfp is expressed in $A Q R, P Q R$, and URXL R. Bar, $10 \mu \mathrm{M}$. and cell death as QL.a while migrating anteriorly, with AQR completing migration near the posterior pharyngeal bulb in the head (Figure 1) (Maloof et al. 1999; Whangbo and Kenyon 1999). Initial Q migrations are controlled autonomously by the receptor molecules UNC-40/DCC and PTP-3/LAR (Honigberg and Kenyon 2000; Sundararajan and Lundquist 2012) and nonautonomously by the Fat-like cadherin CDH-4 (Sundararajan et al. 2014). Later Q-descendant migrations are controlled by Wnt signaling (Whangbo and Kenyon 1999; Zinovyeva and Forrester 2005; Zinovyeva et al. 2008; Harterink et al. 2011), which appears to not be involved in initial migration (Josephson et al. 2016a), and by the transmembrane receptor MIG-13 in parallel with SDN-1/Syndecan (Wang et al. 2013; Sundararajan et al. 2015).

In this work, a forward genetic screen was used to identify additional molecules that regulate $\mathrm{Q}$ migrations. This screen identified an allele of $n f m-1$, which encodes a C. elegans neurofibromatosis type II (NF2)/Merlin molecule. NF2 acts as a tumor suppressor in humans, and mutations in the gene lead to development of neurofibromatosis type II (Gusella et al. 1996; Gutmann et al. 1997), a disease of benign schwannomas. $\mathrm{NF} 2 /$ Merlin is involved in signaling pathways involving hippo, mTOR, and PI3K-Akt (Zhao et al. 2007; Striedinger et al. 2008; James et al. 2009; Okada et al. 2009). Additionally, NF2 is involved in nervous system maintenance, corpus callosum development, and axon guidance (Schulz et al. 2013, 2014; Lavado et al. 2014). In corpus callosum development, NF2 inhibits the hippo pathway component Yap. In NF2 mutants, this inhibition is relieved, resulting in increased expression of Slit2, a secreted axon guidance cue that prevents midline crossing. This leads to defects in midline crossing of axons in the callosum (Lavado et al. 2014).

Here we report that $n f m-1$ mutants display AQR and PQR migration defects. Mosaic analysis and expression studies indicated that NFM-1 does not act in the Q cells themselves but rather nonautonomously, and possibly in muscle. Finally, we show a genetic interaction between NFM- 1 and the secreted guidance cue SLT-1 in AQR migration. In vertebrates, Slit1 and Slit2 are required for guidance of many axons, acting through the Robo family receptors (Nguyen Ba-Charvet et al. 1999; Piper et al. 2000; Bagri et al. 2002; Unni et al. 2012; Kim et al. 2014). The Slit-Robo guidance pathway is conserved in C. elegans, where SLT-1 acts as a guidance cue for several neurons through SAX-3/Robo (Hao et al. 2001; Chang et al. 2006; Quinn et al. 2006; Xu and Quinn 2012). In general, detection of extracellular guidance cues such as Slit cause cytoskeletal changes that result in directed migration of cells and axonal growth cones, most typically repulsion. We show that slt-1 mutations enhance AQR migration defects of $n f m-1$ mutations, and that $s a x-3$ mutants display defects in $\mathrm{AQR}$ and $\mathrm{PQR}$ migration. In sum, results presented here are consistent with a model in which NFM-1 regulates AQR and $\mathrm{PQR}$ migration by controlling the production of an extracellular cue that might act with SLT-1 or in parallel to SLT-1 to promote $\mathrm{Q}$ migrations.

\section{Materials and Methods}

\section{Nematode strains and genetics}

C. elegans were grown under standard conditions at $20^{\circ}$ on nematode growth media (NGM) plates (Sulston and Brenner 1974). N2 Bristol was the wild-type strain. Alleles used include LG III: $n f m-1$ (ok754), nfm-1(lq132) and LG X: sax3(ky123), slt-1(ok255), slt-1(eh15). The Pslt-1::gfp transgene kyIs174 was used (Hao et al. 2001). nfm-1(ok754) was maintained as a balanced heterozygote over the $h T 2$ balancer (nfm-1 (ok754)/hT2). Standard gonadal injection was used to create the following extrachromosomal arrays:

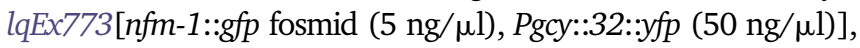



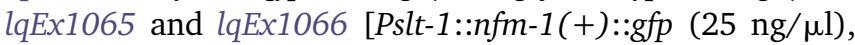

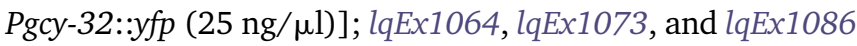


Ultraviolet trimethylpsoralen (UV/TMP) techniques (Mello 
and Fire 1995) were used to integrate extrachromosomal arrays to generate the following transgenes: LGII: lqIs244


location lqIs247 [lqEx773, nfm-1::gfp], lqIs274 [lqEx834,

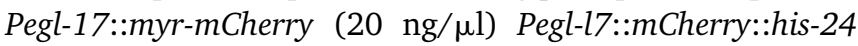
$(20 \mathrm{ng} / \mu \mathrm{l})]$. The $n f m-1:: g f p$ fosmid with $g f p$ fused to the end of the $n f m-1 A$ isoform was obtained from the TransgeneOme project, clone 7039520022144752 D02 (Sarov et al. 2006). $n \mathrm{fm}$-1 (ok754) was maintained as a heterozygote over the $h T 2$ balancer because homozygous ok754 animals arrest during larval stages, but positions of AQR and PQR could be scored in these arrested animals. Genotypes with $\mathrm{M}+$ had maternal contribution from the $h \mathrm{~T} 2$ balancer.

\section{Forward genetic screen for $A Q R$ and $P Q R$ migration defects}

Late L4 hermaphrodites were treated with ethyl methanesulfonate (EMS) mutagen as previously described (Sulston and Hodgkin 1988). These animals were allowed to self-fertilize, and three $\mathrm{F}_{1}$ hermaphrodites were placed on plates (three/ plate). The $F_{2}$ progeny of these hermaphrodites were screened using a fluorescence dissecting microscope, and animals with $\mathrm{AQR}$ and $\mathrm{PQR}$ migration defects were selected. Only one new mutant per $F_{1}$ plate was retained to ensure independent events. This screen identified lq132. The genome of the lq132-bearing strain LE3406 was sequenced, and variants were annotated using the Cloudmap protocol (without Hawaiian strain mapping) (Minevich et al. 2012). lq132 was outcrossed to N2 at least three times.

\section{Scoring Q-cell and $A Q R / P Q R A Q R$ migration}

To score Q-cell protrusions, animals with expression of GFP in the $\mathrm{Q}$ neuroblasts from the egl-17 promoter (ayIs9) were synchronized to 1-2.5 hr posthatching (Chapman et al. 2008; Sundararajan and Lundquist 2012). Briefly, gravid adults were allowed to lay eggs overnight. Plates were washed with M9 buffer, and eggs remained attached to plate. Hatched larvae were collected every half hour using M9 washes and placed onto clean NGM plates for later imaging. Protrusion length was quantified from front of cell body to leading edge of protrusions in ImageJ, with significance determined by a $t$-test. AQR migrates to a position near postdeirid ganglia in the region of the posterior pharyngeal bulb, and PQR migrates posteriorly to a position near the phasmid ganglia posterior to the anus. We used a method as described previously to score AQR and PQR position using Pgcy-32 to drive expression of fluorescent proteins (Shakir et al. 2006; Chapman et al. 2008). Five positions in the anterior-posterior axis of the animal were used to score $A Q R$ and $P Q R$ position. Position 1 was the wild-type position of $A Q R$ and is the region around the posterior pharyngeal bulb. Neurons anterior to the posterior pharyngeal bulb were not observed. Position 2 was posterior to position 1, but anterior to the vulva. Position 3 was the region around the vulva, position 4 was the birthplace of $Q$ cells, and position 5 was posterior to the anus, the wild-type position of PQR (see Figure 2D). A Leica
DM550 equipped with YFP, CFP, GFP, and mCherry filters, was used to acquire all micrographs, and for visualization of $\mathrm{A} / \mathrm{PQR}$ for scoring. Micrographs were acquired using a Qimaging Retiga camera. Significances of difference were determined using Fisher's exact test.

\section{Mosaic analysis}

Mosaic analysis was conducted as previously dscribed (Chapman et al. 2008; Sundararajan et al. 2014) and involved generating a rescuing extrachromosomal array carrying $n f m-1(+)$, and an independent marker of $A Q R$ and $P Q R$ position. The positions of $A Q R$ and $P Q R$ were determined in mosaics in which the rescuing extrachromosomal array was lost in AQR and/or PQR.

A rescuing $n f m-1(+)$ extrachromosomal array was generated using the $n f m-1:: g f p$ fosmid with a Pgcy-32::yfp marker (lqEx773). This array was crossed into $n f m-1$ (ok754)/hT2; lqIs58 (Pgcy-32::cfp) to create the rescuing array lqEx773, referred to as $n f m-1(+)$. Presence of the rescuing array was determined by Pgcy-32::yfp expression, and position of AQR and $\mathrm{PQR}$ was determined by Pgcy-32::cfp expression. $n f m-$ 1(ok754)III; $n f m-1(+)$ animals were viable and fertile and had wild-type $A Q R$ and $P Q R$ position, indicating rescue of $n f m-1$ (ok754). Presence of YFP in AQR or PQR indicated $n f m-1(+)$ was present in those cells during their migrations. Pgcy-32 is also expressed in URX, and presence of YFP in the URX neurons indicates other tissues have inherited $n f m-1(+)$. Animals that lost $n f m-1(+)$ in AQR or PQR, and retained $n f m$ $1(+)$ in the other $\mathrm{Q}$ descendant (PQR and $\mathrm{AQR}$, respectively) and $\mathrm{URX}$ were scored for $\mathrm{AQR}$ and $\mathrm{PQR}$ position.

\section{Synchronization of $L 1$ larvae for expression analysis}

L1 animals carrying Pnfm-1::gfp, the $n f m-1:: g f p$ fosmid, and Pslt-1::gfp were synchronized as described above in Scoring $Q$-cell and $A Q R / P Q R A Q R$ migration to the time of $Q$ cell migration (3-5 hr posthatching). Pegl-17::mCherry was used as a Q-cell marker to determine overlapping expression of $n f m-1$ expression constructs.

\section{Data availability}

The authors state that all data necessary for confirmation of the conclusions discussed in the article are represented fully within the article.

\section{Results}

\section{nfm-1 mutants have defective $A Q R$ and $P Q R$ migration}

To identify genes required for $\mathrm{AQR}$ and $\mathrm{PQR}$ migration, a forward genetic screen was conducted (see Materials and Methods). This screen identified the new mutation lq132. The genome of the lq132-bearing strain was sequenced and variants were detected using Cloudmap (Minevich et al. 2012). The strain contained a splice donor mutation after the fifth exon in the $n f m-1$ gene (Figure 2A) (GTATGTGT to ATATGTGT). To determine whether $n f m-1$ mutation in the $l q 132$ strain caused 
A


$N>100$

${ }^{* *} p<0.005, * * * p<0.0005$ compared to wild-type

$\# \# p<0.005, \# \# \# p<0.0005$ compared to corresponding $n f m-1$ mutant
B

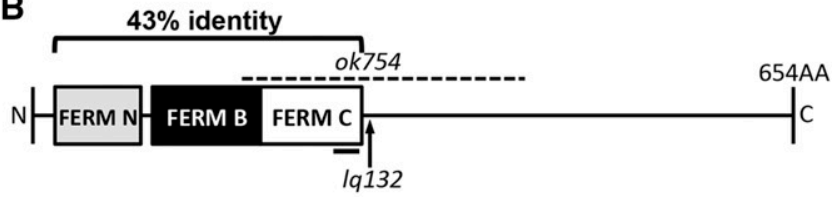

D



$\mathbf{F}$

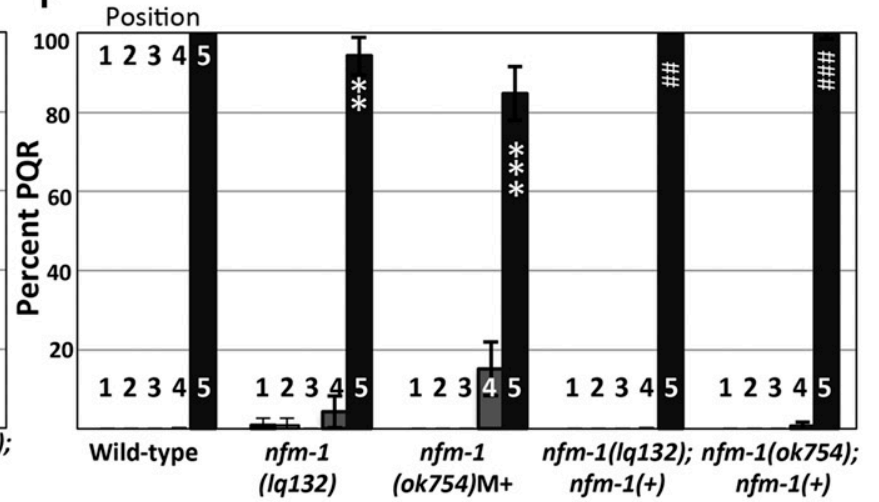

Figure 2 Position of Q descendants AQR and PQR in $n f m-1$ mutants. (A) Diagram of the $n f m-1$ locus and alleles used. The ok754 deletion (dashed line) and Iq132 splice site mutation (arrow) are noted. The alternate $3^{\prime}$ exon use in the three $n f m-1$ isoforms A-C are shown (from WormBase). (B) NFM-1 isoform A domain structure and allele locations are shown. The FERM domain lobes N (gray), B (black), and C (white) are shown. The black bar under FERM C represents predicted actin-binding motif. The dashed line is ok754 in-frame deletion, and /q132 splice donor mutation location is marked by an arrow. NF2 and NFM-1 show 43\% identity throughout the FERM N, B, and C regions. (C) Merged DIC and fluorescent micrograph of an $n f m-1$ (ok754) arrested larval mutant animal. Both $\mathrm{AQR}$ and $\mathrm{PQR}$ failed to migrate (PQR wild-type position noted by arrowhead). Bar, $10 \mu \mathrm{M}$. (D) Diagram of scoring positions in an L4 animal used in E and F, with wild-type locations of $A Q R$ and $P Q R$ shown as magenta circles. (E and F) Chart showing percent of $A Q R$ (E) or PQR (F) in positions 1-5 in different genotypes as shown in D. All animals unless otherwise noted were scored using lqls58 (Pgcy-32::cffp). M+ indicates animals were scored from heterozygous mother and have wild-type maternal contribution of $n f m-1$. nfm-1(+) animals harbor the array containing the $n f m-1:: g f p$ fosmid. Asterisks indicate degree of significance of difference from wild-type $(N>100 ; * P<0.05, * * P<0.005, * * *$ $P<0.0005$, Fisher's exact test). Pound signs indicate, for that position, a significant rescue of corresponding $n f m-1$ mutant $(N>100$; \# $P<0.05$, \#\# $P<$ 0.005, \#\#\# $P<0.0005$, Fisher's exact test). Error bars represent two times the SE of the proportion in each direction.

$\mathrm{AQR}$ and $\mathrm{PQR}$ defects, we scored $\mathrm{AQR}$ and $\mathrm{PQR}$ migration in a second allele, the existing the $n f m-1$ (ok754) mutant generated by the $C$. elegans gene knockout consortium. nfm-1 (ok754) is an in-frame 1042-bp deletion with breakpoints in exons 3 and 7 that removes all of exons 4-6 (Figure 2, A and B). $n f m-1$ (ok754) homozygotes arrested as larvae, but we were able to score $\mathrm{AQR}$ and PQR position in arrested larvae. $n \mathrm{fm}-1$ (ok754) had strong AQR defects (Figure 2, C and D), with $88 \%$ of AQR failing to migrate to the head, and occasional (1\%) posterior AQR migration (Figure 2, C and E). nfm-1 (ok754) also had significant PQR defects, with $15 \%$ of $\mathrm{PQR}$ failing to migrate into the wildtype position 5, posterior to the anus (Figure $2 \mathrm{~F}$ ). To confirm that $n f m-1$ was the causative locus, we found that an $n f m-1:: g f p$ fosmid transgene rescued AQR and PQR defects of both lq132 and ok754 mutants (Figure 2, E and F). $n f m-1$ encodes a protein similar to human NF2/Merlin, and contains Four-Point-One Ezrin Radixin and Moesin (FERM) $\mathrm{N}, \mathrm{B}$, and $\mathrm{C}$ domains at the $\mathrm{N}$ terminus (Figure 2B). Three isoforms of $n f m-1$ are predicted, differing at the $3^{\prime}$ end (WormBase) (Figure 2A). lq132 and ok754 are predicted to affect all three isoforms. The functional differences, if any, between these isoforms are not known.

The lq132 splice donor mutation occurred after the conserved FERM domain regions, and the ok754 in-frame deletion removes the entire FERM $C$ domain, including the putative actin-binding site (Figure 2B). RNAi of $n f m-1$ caused embryonic lethality (Skop et al. 2004). Thus, lq132 is likely a hypomorphic mutation and retains some function. ok754 mutants have wild-type maternal contribution, which might allow the animals to bypass embryonic lethality and arrest later as larvae. It is also possible that the 

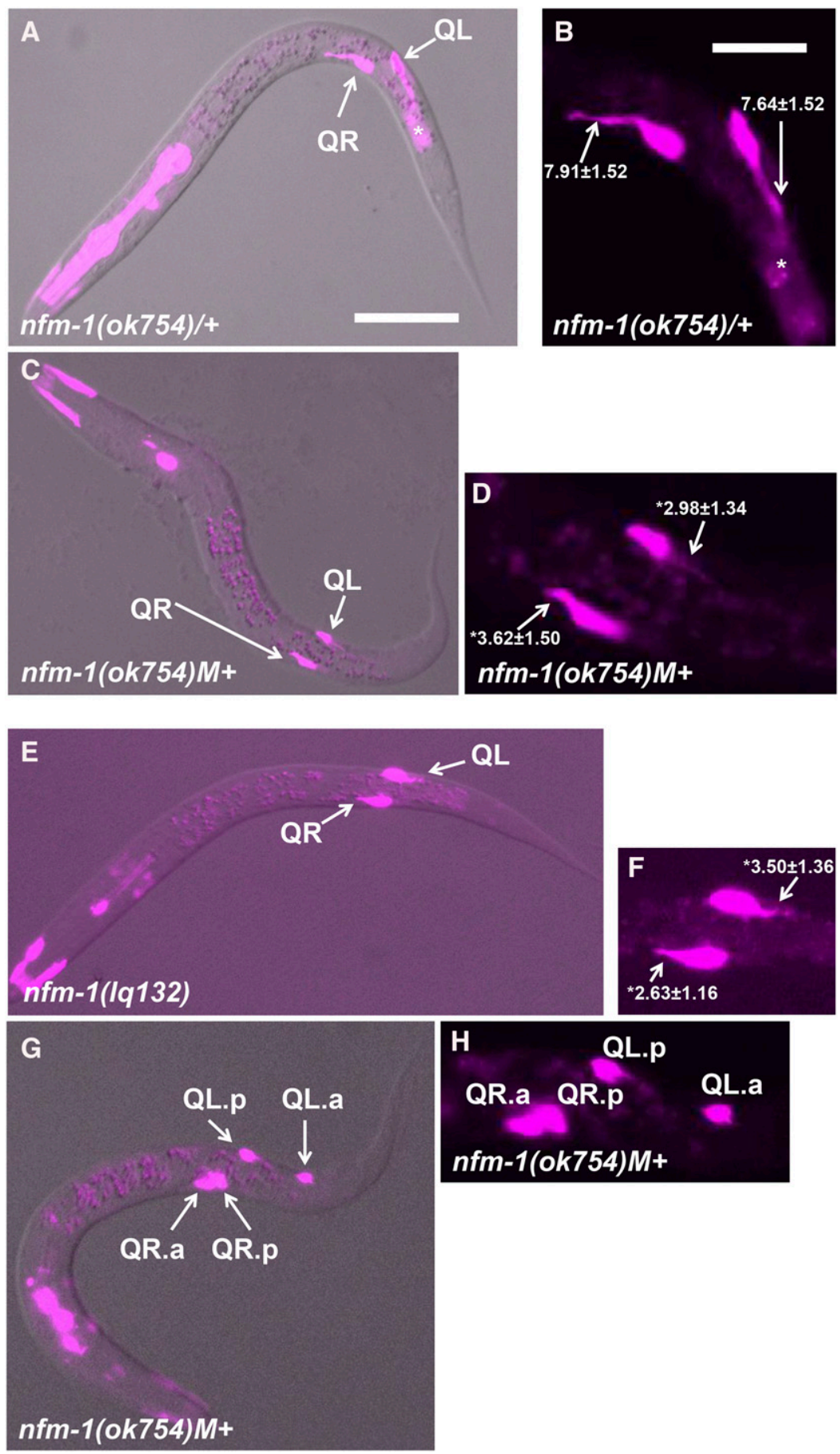

Figure 3 Early Q migrations in $n f m-1$ mutants. Fluorescent micrographs of L1 animals with ayls9[egl-17::gfp] expression are shown (magenta). Anterior is to the left. $(A, C, E$, and $F)$ Merged with a differential interference contrast image. ( $B, D, F$, and $G$ ) Enlarged images of the migrating $Q$ cells. The average length and SE of $Q$ protrusions in micrometers are indicated in $\mathrm{B}, \mathrm{D}$, and $\mathrm{F}(n=20$ in all cases). The asterisks indicate statistical significance compared to wild type ( $t$-test; $P<0.0001)$. (A and $B$ ). Wild-type $\mathrm{Q}$ cells display robust protrusions at $1-2.5 \mathrm{hr}$ posthatching (arrows). This animal is a balanced $\mathrm{nfm}$ 1(ok754)/hT2 heterozygote with one wild-type copy of $\mathrm{nfm}-1$. The bright fluorescence in the anterior is $\mathrm{gfp}$ expression in the pharynx associated with the $h T 2$ balancer chromosome, and the fluorescence posterior to the Q cells is background associated with the ayls9[Pegl-17::gfp] transgene (asterisk). (C and D). An nfm-1(ok754) homozygote with wild-type maternal contribution $(\mathrm{M}+)$ at $1-2.5 \mathrm{hr}$ posthatching displays Q cells with shortened protrusions compared to wild-type (arrows). (E and F). An nfm1(lq132) homozygote at 1-2.5 hr posthatching displays shortened protrusions (arrows). ( $\mathrm{G}$ and $\mathrm{H}$ ). An $\mathrm{nfm}$ 1(ok754)M+ animal at 6-7 hr posthatching. QL.p has migrated posteriorly, whereas both QR.a and QR.p have failed to migrate anteriorly and remain near their birthplace. Bars, $10 \mu \mathrm{m}$ for $A, C, E$, and $F$ and $5 \mu \mathrm{m}$ for $B, D, F$, and $\mathrm{G}$. ok754 in-frame deletion retains some function. AQR migration defects in ok754 were significantly stronger than lq132 $(P<$ 0.001), suggesting that ok754 is a stronger allele than lq132.

\section{NFM-1 is required for Q-cell protrusion and migration}

A Pegl-17::gfp transgene was used to inspect early Q migration (Branda and Stern 2000; Cordes et al. 2006; Josephson et al. 2016a). Between 1 and $2.5 \mathrm{hr}$ after hatching, Q cells in wild-type extend robust protrusions over the neighboring seam cells in their direction of eventual migration (QR to the anterior over V4, and QL to the posterior over V5) (Figure 3, A and
B) (Chapman et al. 2008). In $\mathrm{nfm}$-1(ok754) $\mathrm{M}+$ and $\mathrm{nfm}$ 1(lq132) homozygotes, Q-cell protrusions at 1-2.5 hr were significantly shorter than in wild type (Figure 3, C-F). No defects were observed in the direction of protrusion. These data indicate that NFM-1 is required for robust Q-cell protrusion. Between 3 and $3.5 \mathrm{hr}$ after hatching, wild-type Q-cell bodies migrate atop the neighboring seam cells, and the first $\mathrm{Q}$ cell division occurs between 4 and $4.5 \mathrm{hr}$ (Chapman et al. 2008). Despite reduced protrusions in $n f m-1$ mutants, the $Q$ cells completed their anterior and posterior migrations before division ( $n>20$ for both ok754 and lq132). 

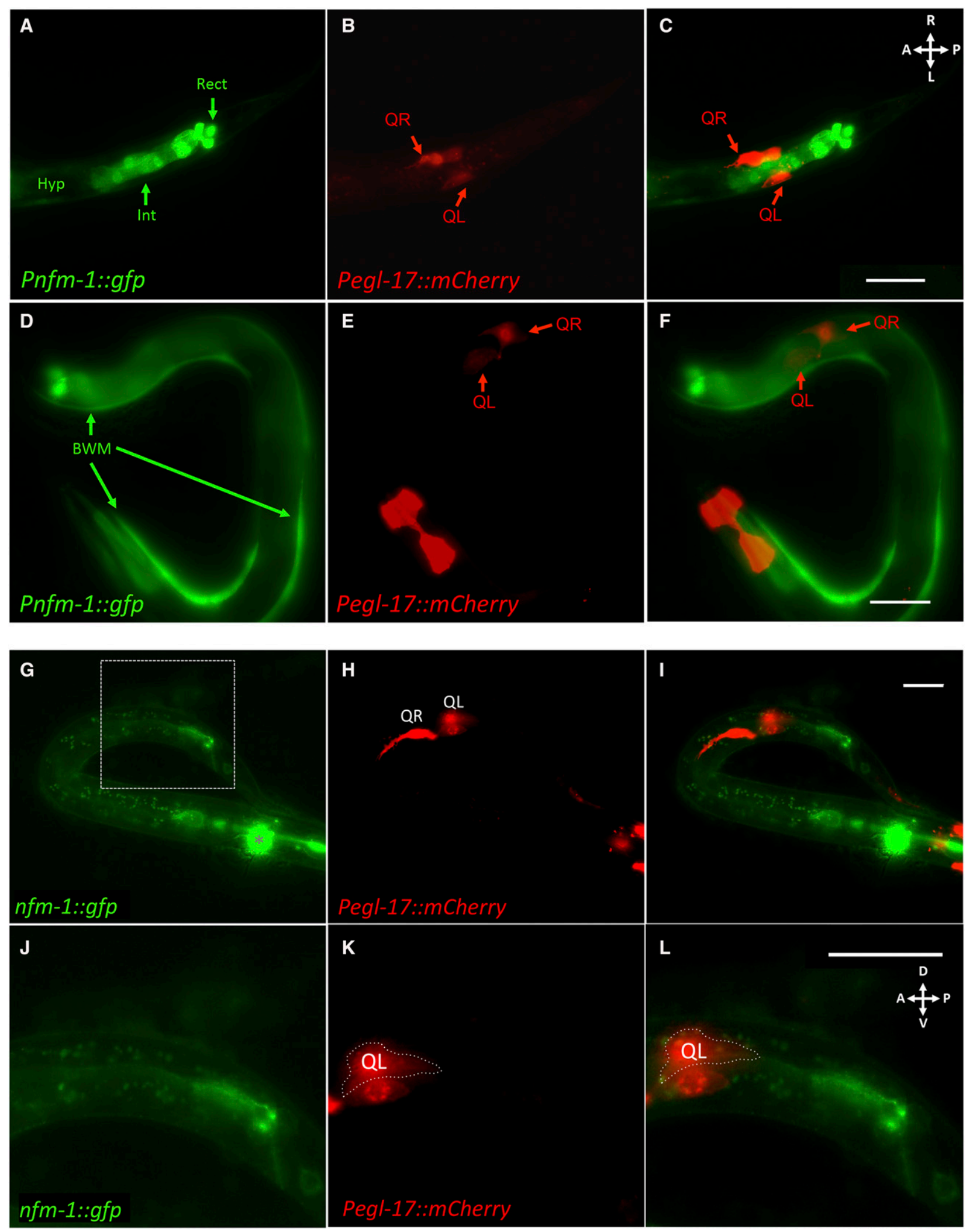

Figure $4 \mathrm{nfm}-1$ was not expressed in Q cells during their early migrations. (A-C) Ventral view of the posterior region of a Pnfm-1::gfp; Pegl-17::mCherry transgenic animal staged to 3-3.5 hr posthatching. (A) GFP micrograph showing expression of Pnfm-1::gfp. Expression was seen in posterior cells near the anus, including posterior intestinal cells (Int) and the three rectal gland cells (Rect). Other unidentified cells in the region were possibly the anal sphincter muscle and the stomatointestinal muscle. Variable hypodermal expression was observed along the length of the animal (Hyp). (B) An mCherry micrograph shows Q-cell-specific expression during their migrations. (C) Merged. GFP is not observed in Q cells, but is expressed in neighboring tissues 
After division at 4-4.5 hr, the wild-type QR daughters QR.a and QR.p extend anterior protrusions and begin anterior migration, whereas the QL daughters QL.a and QL.p remain rounded and nonpolarized and do not migrate (Josephson et al. 2016a). At 5-7.5 hr after hatching, QL.a migrates posteriorly past QL.p (Josephson et al. 2016a). In $n f m-1$ (ok754)M ,$+ 2 / 20$ QR.a/p daughters failed to migrate anteriorly and stayed near their birthplace, even after QL.a had migrated posteriorly (Figure 3, G and $\mathrm{H}$ ). This defect was not observed in the weaker $n f m$-1(lq132) mutant, although more subtle defects in migration might have escaped detection. Failure of QR.a/p migration might explain the strong AQR migration defects observed in $n f m-1$ (ok754), as AQR is a descendant of QR.a. These data suggest that NFM-1 is required for Q-cell and descendant protrusion and migration. Direction of protrusion and migration was not affected in $n f m-1$ mutants. However, as both mutants likely retain some $\mathrm{nfm}-1$ function, a role of NFM1 in controlling direction of protrusion cannot be excluded.

\section{nfm-1::gfp transcriptional and translational reporter expression was not apparent in $Q$ lineages}

A Pnfm-1::gfp transcriptional reporter was created by using a $2.1-\mathrm{kb}$ region upstream of $n f m-1$ to drive expression of $g f p$. This 2.1-kb region was the entire upstream region between $n f m-1$ and the next gene anmt-2. At the time of $\mathrm{Q}$ migration, this construct showed expression in posterior cells near the anus, including posterior intestinal cells, the three rectal gland cells, and other unidentified cells that might be the anal sphincter muscle and the stomatointestinal muscle (Figure 4, A-C). Variable expression in the hypodermis was also observed (Figure 4, A-C), as well as in body wall muscle cells (Figure 4, D-F). Pnfm-1::gfp expression was not observed in migrating Q neuroblasts (Figure 4, A-C).

Full-length NFM-1::GFP expression from the rescuing fosmid was not observed in migrating Q cells (Figure 4, G-L). NFM-1::GFP was detected in the posterior gut region. Three isoforms for $n f m-1$, differing at the $3^{\prime}$ end, are reported (WormBase). This fosmid contains the $g f p$ tag at the end of the $n f m-1 A$ isoform and so will not report the expression of the $B$ and $C$ isoforms. We do not know which isoforms are required for $\mathrm{AQR}$ and $\mathrm{PQR}$ migration, but the $n f m-1$ promoter was not active in $\mathrm{Q}$ cells, and $n f m-1 A$ isoform expression was not observed in the $\mathrm{Q}$ cells.

\section{Mosaic analysis suggests a nonautonomous requirement for $n f m-1$ in anterior $A Q R$ migration}

No expression of $n f m-1$ was observed in migrating $Q$ neuroblasts. Genetic mosaic analysis using a rescuing $n f m-1(+)$ extrachromo- somal array was used to test whether $n f m-1$ was required in the $\mathrm{Q}$ cells themselves for proper $\mathrm{AQR}$ and $\mathrm{PQR}$ migration (see Materials and Methods). In C. elegans, extrachromosomal arrays are not stably inherited mitotically and can be lost during cell divisions, creating genetically mosaic animals. We used an established strategy to score mosaic animals that had lost an $n f m-1$ (+) rescuing transgene in AQR or PQR lineage (see $M a-$ terials and Methods and Chapman et al. 2008; Sundararajan et al. 2014). This strategy uses a stable $P g c y$-32::cfp integrated transgene to visualize $\mathrm{AQR}$ and $\mathrm{PQR}$ in all animals, and an unstable array carrying the rescuing $n f m-1:: g f p$ fosmid and Pgcy-32::yfp, which we refer to as $n f m-1(+)$. The AQR, PQR, and URX cells are derived from well-separated lineages, with URX and QR/QL lineages distinguished after the second embryonic division, and the QL and QR lineages after the third (Figure 5A), making mosaic animals with losses in specific lineages readily identifiable (Figure $5, \mathrm{~B}$ and $\mathrm{C}$ ).

$n f m-1$ (ok754) animals that harbored the $n f m-1(+)$ array were viable, fertile, and were rescued for $\mathrm{AQR}$ and $\mathrm{PQR}$ migration (Figure 2, E and F). We analyzed 89 mosaic animals in which the $n f m-1(+)$ array was lost from the AQR lineage, but retained in PQR and URX lineages as shown in Figure 5, B and C. These animals were rescued for AQR migration defects despite loss of $n f m-1(+)$ in AQR compared to $n f m-1$ (ok754) alone (Figure $5 \mathrm{D}$ ), suggesting that $n f m-1$ is required nonautonomously for anterior AQR migration. Similarly, PQR migration defects were still rescued in 75 mosaic animals in which $\mathrm{PQR}$ had lost the $n f m-1(+)$ array (Figure 5E). Loss of $n f m-1(+)$ in AQR or PQR rescued $n f m-1$ (ok754) defects to a similar level as in animals in which no loss occurred [nfm-1(+) in AQR and PQR] (Figure 5, $\mathrm{D}$ and $\mathrm{E})$. It is possible that perdurance of NFM-1 protein, or array loss in the $\mathrm{Q}$ lineages themselves, led to $n f m-1$ function in the $\mathrm{Q}$ lineages despite loss in $\mathrm{AQR}$ or $\mathrm{PQR}$. To account for these rare but possible events, we scored at least 70 mosaic animals. Overall, mosaic analysis suggests that $n f m-1$ acts nonautonomously for $\mathrm{AQR}$ and $\mathrm{PQR}$ migration, as loss of the rescuing array in AQR or PQR did not correlate with mutant phenotype.

\section{Expression of $n f m-1$ in muscles rescued $A Q R$ and $P Q R$ migration defects}

$n f m-1(+)$ expression was driven from two promoters with expression in muscles, the slt-1 and myo-3 promoters. At the time of $\mathrm{Q}$ protrusion and migration, the slt-1 promoter was active in dorsal- and ventral-posterior body wall muscles (Figure 6, A-C) (Hao et al. 2001). It was also expressed in cells in the head and the anal sphincter muscle as previously reported (Figure 6, A-C) (Hao et al. 2001). Pslt-1 expression was not observed in protruding and migrating $\mathrm{Q}$

and posterior cells. Bar, $10 \mu \mathrm{m}$ for A-C. (D-F) An L1 animal 3-3.5 hr posthatching with Pnfm-1::gfp expression in body wall muscles. Bar, 20 um for D-F. (G-L) Lateral view of a staged 3-3.5 hr posthatching L1 with full length $n f m-1:: g f p$ and Pegl-17::mCherry expression. (G) Fluorescent micrograph of GFP expression from $n f m-1:: g f p$ rescuing fosmid. Asterisk marks URX expression of Pgcy-32::yfp in the head that was not excluded by GFP filter. The dashed rectangle indicates the enlarged posterior section in J-L. (H) Pegl-17::mCherry, fluorescent micrograph showing location of early Q neuroblasts. QL is out of focus because QL and QR are on different planes, QR on the right side and QL on left side of the animal. (I) Merge of A and B. No overlap of mCherry and GFP was observed. (J-L) Enlarged posterior section of G-I. (J) Enlargement of A to show nfm-1::gfp present in posterior region near the anus. (K) Enlargement of B. QL is outlined to distinguish it from the V5 seam cell that transiently expresses Pegl-17. (L) Enlargement of C. Bar, $10 \mu \mathrm{m}$. In all micrographs, anterior is to the left. 
A

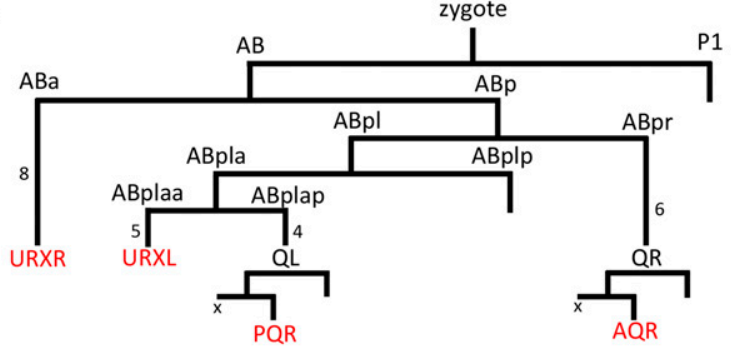



E

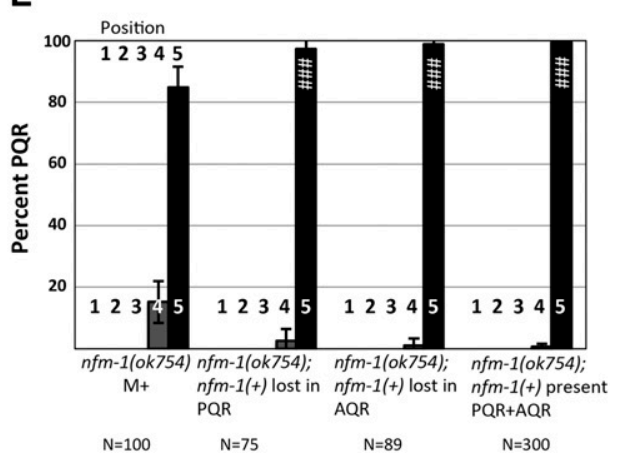

Figure $5 \mathrm{nfm}-1$ mosaic analysis. (A) The abbreviated lineage of cells that express Pgcy-32 (red). Numbers next to lines indicate the number of cell divisions not shown. The $X$ next to $A Q R$ and $P Q R$ indicates the sister of $A / P Q R$ (QL/R.aa) that undergoes programmed cell death. (B) Fluorescent micrograph taken with CFP filter of $n f m-1(0 k 754)$; nfm-1(+), Pgcy-32::cfp mosaic animal with correct placement of $A Q R$ and PQR. (C) Fluorescent micrograph of the same animal from $B$ using a YFP filter. AQR is not visible in this animal, indicating that somewhere in the $A Q R$ lineage, the $n f m-1(+)$ transgene was lost. YFP is detected in $U R X L / R$, and $P Q R$ indicating many tissues retained $n f m-1(+)$. Bar, $10 \mu \mathrm{M}$. ( $D$ and $E$ ) Quantification of $A Q R(A)$, and $P Q R(B)$ migration as in Figure 2, with $n f m-1(+)$ mosaic animals. $n f m-1(+)$ represents presence of $n f m-1$ rescuing fosmid. $n f m-1(+)$ rescued ok754 lethality, and animals were maintained as rescued homozygous ok754 mutants. Mosaic animals have $\mathrm{nfm}$ $1(+)$ in URX but have lost $n f m-1(+)$ in either $\mathrm{AQR}$ or $\mathrm{PQR}$. Pound signs indicate, for that position, a significant rescue of corresponding $\mathrm{nfm}-1$ mutant $(N>100$; $\#<0.05$, \#\# $P<0.005$, \#\#\# $P<0.0005$, Fisher's exact test). Error bars represent two times the SE of the proportion. neuroblasts (Figure 6, A-C). The entire $n f m-1 A$ coding region fused to $g f p$ was placed under the control of Pslt-1. Two independent lines of $n f m-1$ (ok754)M+ animals harboring Pslt$1:: n f m-1(+)$ still arrested as early larvae but were significantly rescued for AQR migration defects (Figure 6D). PQR defects were not significantly rescued by Pslt-1::nfm-1(+).

The myo-3 promoter is expressed in body wall muscles, the vulval muscles, and the anal sphincter muscle (Okkema et al. 1993). Two independent lines of Pmyo-3::nfm-1(+)::gfp rescued AQR and PQR defects of $n f m-1$ (ok754)M+ (Figure 6D). These animals also grew to be sterile adults, indicating that Pmyo-3::nfm-1(+)::gfp expression partially rescued the larval arrest of $n f m-1$ (ok754)M+ animals. One line of Pmyo-3::nfm$1(+):: g f p$ also rescued AQR and PQR defects of $n f m-1$ (lq132) (Figure 6D). Expression of slt-1 (Figure 6), myo-3, and $n f m-1$ (Figure 4) overlap in the body wall muscles and possibly the anal sphincter muscle, suggesting that these tissues might be the cellular focus of $n f m-1$ activity in Q migration. Muscle expression of $n f m-1$ only partially rescued AQR and PQR defects, suggesting that $n f m-1$ could be required in other tissues for full rescue.

\section{slt-1 mutations enhance AQR defects of $\mathrm{nfm}-1$ (lq132)}

Previous studies suggested that NF2 can nonautonomously affect axon guidance in the developing mouse brain (Lavado et al. 2014). This guidance mechanism occurs through regulation of Slit2 mRNA levels, suggesting a transcriptional role of NF2 (Lavado et al. 2014). Slit2 is a secreted guidance cue for developing neurons and is detected by the Robo receptor. Because of interactions between Slit2 and NF2, we investigated the interaction of $n f m-1$ and the C. elegans Slit2 homo$\log$ slt-1 in Q-descendant migration. In this study we used one null allele slt-1(eh15), and one strong loss-of-function in-frame deletion allele slt-1(ok255) (Hao et al. 2001; Steimel et al. 2013). slt-1 mutations had no effect on AQR and $\mathrm{PQR}$ migration on their own, but enhanced AQR migration defects of $n f m-1$ (lq132) and $n f m-1$ (ok754) (Figure 7). slt-1 had no effect on PQR migration in double mutants. We tested the SLT-1 receptor SAX-3/Robo, and sax-3(ky123) mutants showed weak but significant defects in both AQR and $\mathrm{PQR}$ migration, consistent with SAX-3 promoting anteriorposterior migration of the $Q$ lineages (Figure 7).

slt-1 was not expressed in protruding and migrating $Q$ neuroblasts (Figure 6, A-C), suggesting a likely nonautonomous effect expected of a secreted signaling molecule. Loss of NF2 in mouse led to increased levels of Slit2 expression (Lavado et al. 2014). We detected no discernible change in Pslt-1::gfp expression in $n f m-1$ (lq132) and $n f m-1$ (ok754)M+ animals at $1-2.5 \mathrm{hr}$ posthatching, including body wall muscle and anal sphincter 

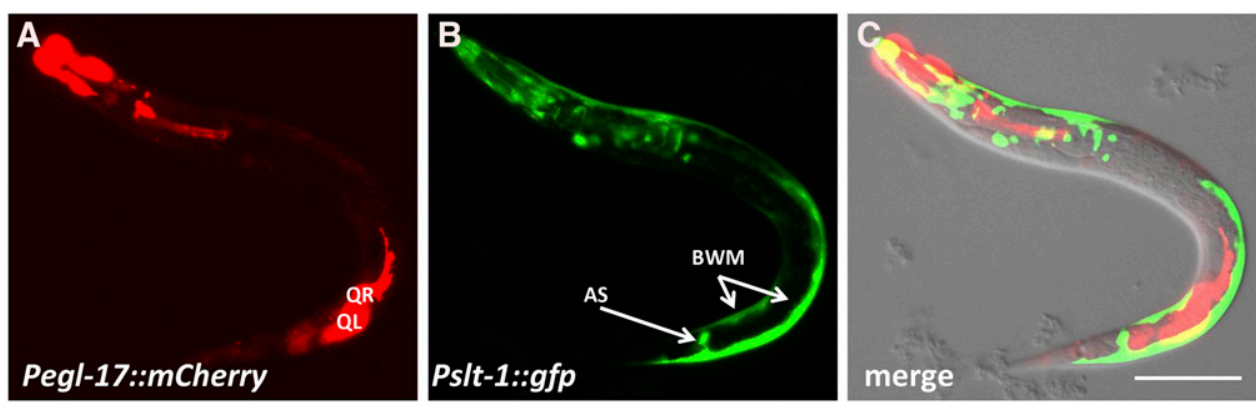

D

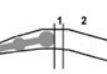

merge

\begin{tabular}{|c|c|c|c|c|c|c|c|c|c|c|}
\hline \multirow[b]{2}{*}{ Genotype $(n=100)$} & \multicolumn{5}{|c|}{ AQR position (\%) } & \multicolumn{5}{|c|}{ PQR position (\%) } \\
\hline & 1 & 2 & 3 & 4 & 5 & 1 & 2 & 3 & 4 & 5 \\
\hline wild-type & 100 & 0 & 0 & 0 & 0 & 0 & 0 & 0 & 0 & 100 \\
\hline$n f m-1(o k 754) M+$ & 13 & 37 & 38 & 11 & 1 & 0 & 0 & 0 & 15 & 85 \\
\hline$n f m-1(o k 754) M+;$ Ex[Pslt-1::nfm-1]\#1 & $75^{1}$ & 18 & 6 & 0 & 0 & 0 & 0 & 0 & 11 & 89 \\
\hline$n f m-1(o k 754) M+; E x[P s l t-1:: n f m-1] \# 2$ & $80^{1}$ & 14 & 6 & 0 & 0 & 0 & 0 & 0 & 8 & 92 \\
\hline$n f m-1(o k 754) M+;$ Ex[Pmyo-3::nfm-1]\#1 & $48^{1}$ & 24 & 28 & 0 & 0 & 0 & 0 & 0 & 1 & $99^{2}$ \\
\hline$n f m-1(o k 754) M+;$ Ex[Pmyo-3::nfm-1]\#2 & $86^{1}$ & 12 & 2 & 0 & 0 & 0 & 0 & 0 & 2 & $98^{3}$ \\
\hline$n f m-1(I q 132)$ & 90 & 7 & 1 & 1 & 1 & 0 & 1 & 1 & 4 & 94 \\
\hline$n f m-1$ (Iq132); Ex[Pmyo-3::nfm-1]\#3 & $98^{4}$ & 2 & 0 & 0 & 0 & 0 & 0 & 0 & 0 & $100^{5}$ \\
\hline
\end{tabular}

$1 p<0.0001$ compared to $n f m-1$ (ok754)M+alone.

${ }^{2} p=0.0140$ compared to $n f m-1$ (ok754) M+ alone.

$p=0.0015$ compared to $n f m-1(o k 754) M+$ alone.

${ }^{4} p=0.0330$ compared to $n f m-1(l a 132)$ alone.

${ }_{5} p=0.0289$ compared to $n f m-1$ (lq132) alone.

muscle (data not shown). However, expression of $n f m-1(+)$ from the slt-1 promoter rescued AQR defects of $n f m-1$ (ok754) $M+$ (Figure 6, D and E), suggesting that $n \mathrm{fm}-1$ and slt-1 might be acting in the same cells to regulate $\mathrm{Q}$ migration.

\section{Discussion}

\section{The NF2/Merlin molecule NFM-1 promotes protrusion and migration of $Q$ cells and their descendants}

Complete migration of the QR and QL descendants AQR and $\mathrm{PQR}$ requires the coordination of many genes (Middelkoop and Korswagen 2014). Although numerous molecules have been identified that act in the Q cells to promote migration, such as the transmembrane receptors UNC-40/DCC, PTP-3/ LAR, and MIG-13 (Sundararajan and Lundquist 2012; Wang et al. 2013; Sundararajan et al. 2015), fewer have been identified that act outside the $\mathrm{Q}$ cells to control their migration. Of the nonautonomous genes that have been implicated in Q-descendant migration, most are secreted molecules such as Wnts (Hunter et al. 1999; Whangbo and Kenyon 1999; Korswagen 2002; Pan et al. 2006) and SPON-1/F-spondin (Josephson et al. 2016b), although the Fat-like cadherin $\mathrm{CDH}-4$ has been demonstrated to nonautonomously affect Q-cell migration (Sundararajan et al. 2014).

Here we present data identifying a nonautonomous role for the FERM domain-containing molecule NFM-1, a predicted cytoplasmic protein, in promoting Q migration. NFM-1 is similar to human NF2/Merlin, the molecule affected in neurofibromatosis type II. We found that mutations in $n f m-1$ resulted in AQR migration defects, and to a lesser extent
Figure 6 Muscle-specific expression of nfm-1 rescues $A Q R$ and PQR defects. (A-C) Expression of Ps/t-1::gfp (kyls174) (Hao et al. 2001) and Pegl-17::mCherry in an L1 animal 3-3.5 hr posthatching. AS, anal sphincter muscle; BWM, body wall muscle. QL and QR are indicated. Bar, $20 \mu \mathrm{m}$ for A-C. (D) Rescue of $n f m$ $1 \mathrm{AQR}$ and PQR defects by cell-specific transgenes. The positions of $A Q R$ and $P Q R$ are as described in Figure 2D. The percentage of cells in each position is indicated, with significance of differences (Fisher's exact test). Two independent Ps/t-1::nfm-1::gfp transgenes rescued $n f m-1$ (ok754) (IqEx1065\#1 and lqEx1066\#2), and three independent Pmyo-3::nfm-1::gfp transgenes rescued $n f m-1$ (ok754) (lqEx1064\#1 and IqEx1086\#2), and $n f m-1$ (Iq132) (IqEx1073\#3).
PQR migration defects. These defects typically manifested as incomplete migrations, suggesting that these $n \mathrm{fm}$ - 1 mutations did not affect direction of migration along the anterior/ posterior axis, but rather the migratory capacity of these cells.

We found that NFM-1 was required for robust protrusion of QR and QL in their initial migrations over V4 and V5, as well as subsequent migration of QR daughters. In no case did we observe $\mathrm{Q}$ protrusion or migration in the wrong direction, suggesting that NFM-1 affects the ability of Q cells to protrude and migrate, but not their direction.

Loss of NF2/Merlin function in either mouse or Drosophila results in embryonic lethality (Fehon et al. 1997; McClatchey et al. 1997). In C. elegans, $n f m-1$ appears to be required in embryonic development similar to other animals, as RNAi against $n f m-1$ is reported as embryonic lethal (Skop et al. 2004), and no null alleles of $n f m-1$ have been described. The two $n f m-1$ mutations studied here likely retain some NFM-1 function. The $5^{\prime}$ splice site mutant $\mathrm{nfm}$-1 (lq132) was viable and fertile, and the in-frame deletion allele $n f m$ 1 (ok754) caused larval arrest possibly due to wild-type maternal contribution. It is possible that complete loss of $n f m-1$ function results in more severe Q-migration defects, possibly even directional defects, not observed in these alleles. The $n f m-1$ (ok754) in-frame deletion removes part of the FERMB domain and the entire FERMC domain, suggesting that these domains are important in $\mathrm{AQR}$ and $\mathrm{PQR}$ migration.

\section{NFM-1 might act in muscles to promote $A Q R$ and PQR migration}

As a cytoskeletal-membrane linker with a potential actin-binding domain, we hypothesized that NFM-1 might regulate actin-based 
A

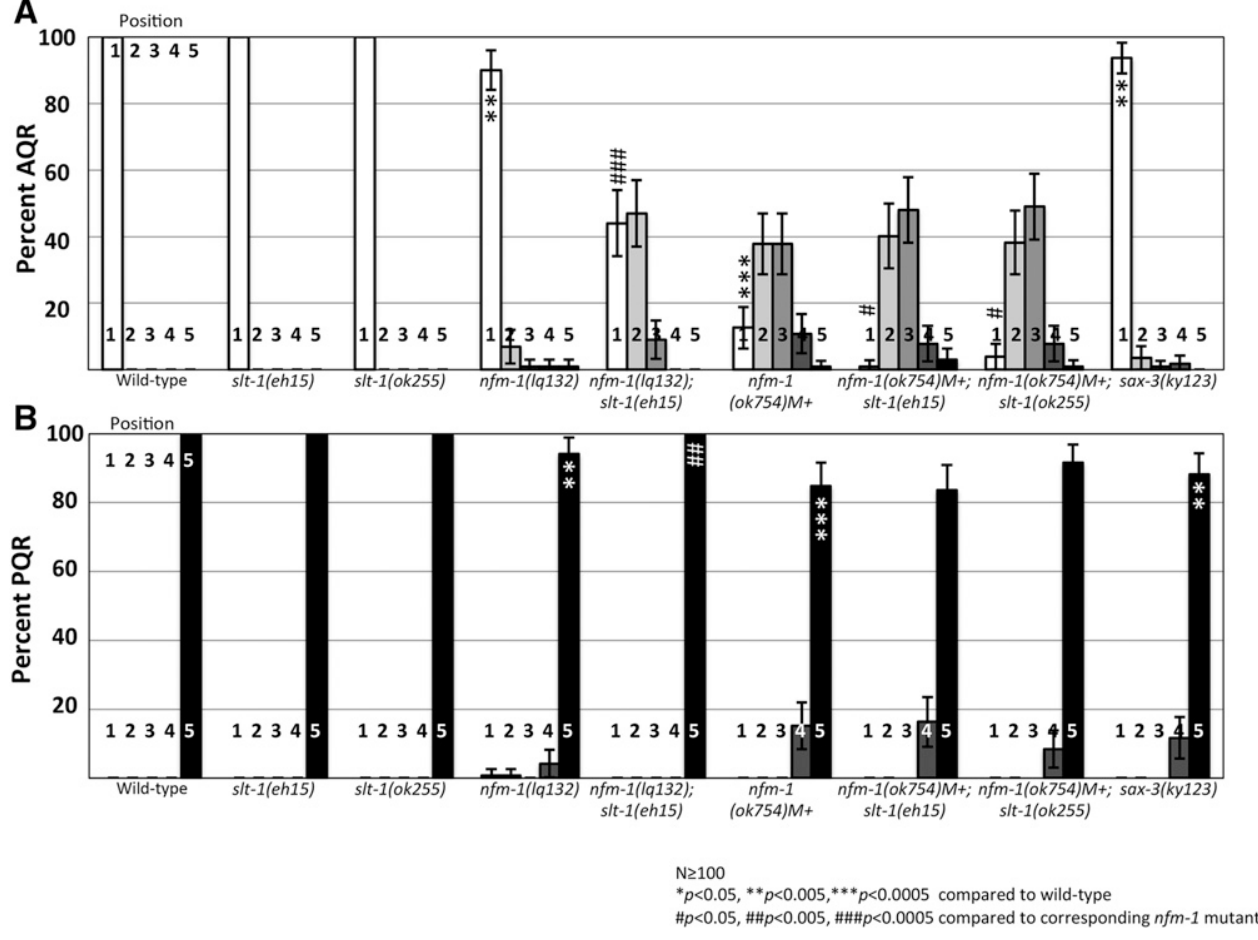

Figure 7 slt-1 enhances $n f m-1$ AQR migration defects. (A) Percentage of AQR in each position, quantified as in Figure 2. (B) $P Q R$ migration. Asterisks indicate significant difference from wild type $(N>100 ; * P<0.05, * P<0.005$, *** $P<0.0005$, Fisher's exact test). Pound signs indicate, for that position, a significant enhancement of the corresponding $n f m-1$ mutant $(N>100$; \# $P<0.05$, \#\# $P<0.005$, \#\#\# $P<$ 0.0005 , Fisher's exact test). Error bars represent two times the SE of the proportion. membrane protrusion in migrating cells. However, no $n f m-1$ expression was observed in the Q cells or descendants, and a genetic mosaic analysis and cell-specific expression indicated that NFM-1 was not required in AQR or PQR for their migration. These data suggest that NFM-1 is required nonautonomously outside of the Q lineages for their migration. Pnfm-1::gfp expression was observed in the posterior region near the anus, including posterior intestine, the rectal gland cells, and potentially the anal sphincter muscle and stomatointestinal muscle. Expression was also noted in body wall muscles. Cell-specific expression of $n f m-1(+)$ from the slt-1 and myo-3 promoters, both expressed in body wall muscles and the anal sphincter muscle, rescued AQR and PQR migration defects of $n f m-1$ (ok754)M+. Thus, $n f m-1$ might be required in body wall muscles and/or the anal sphincter muscle to promote Q migrations. The Q-cell protrusions are in close proximity to the body wall muscle cells as the $\mathrm{Q}$ cells undergo their initial migrations. Interestingly, Pslt-1 expression of $n f m-1$ did not rescue PQR defects, whereas Pmyo-3 expression did. We do not understand the nature of this difference, but it is possible that AQR and PQR have differential requirements for $n f m-1$ expression, either different levels of expression or expression from different tissues.

\section{nfm-1 and slt-1 interact genetically to promote anterior AQR migration}

In Drosophila and mice, NF2/Merlin is known to regulate several signaling pathways, including stimulating the Hippo pathway to inhibit the Yorkie transcription cofactor (Hamaratoglu et al. 2006; Moroishi et al. 2015). In mice, loss of NF2 in neural progenitor cells results in upregulation of Yap (Lavado et al. 2014). High Yap activity leads to ectopic levels of the secreted guidance cue Slit2, which causes defects in midline axon guidance (Lavado et al. 2014). Interestingly, this is a nonautonomous role of NF2 in midline axon guidance, similar to our observation of $n \mathrm{fm}-1$ in $C$. elegans neuronal migration. The Hippo pathway in C. elegans is poorly conserved (Hilman and Gat 2011), although C. elegans YAP-1 is similar to Yap (Iwasa et al. 2013). A role of yap-1 in AQR and PQR migration was not determined. However, $n f m-1$ mutants had no discernible effect on the expression of Pslt-1::gfp, suggesting that NFM-1 does not regulate SLT-1 expression, at least at the transcriptional level.

We tested the role of the single $C$. elegans Slit gene slt- 1 in AQR/PQR migration and interaction with $n f m-1$. slt-1 regulates the anterior-posterior migration of the CAN neurons in embryos (Hao et al. 2001). Although no migration defects were detected in slt-1 mutants alone, they did enhance AQR migration defects of $n f m-1$ (lq132) and $n f m-1$ (ok754). This enhancement is consistent with NFM- 1 and SLT- 1 acting in parallel pathways, but since we do not know the null phenotype of NFM-1 with regard to AQR and PQR, the possibility that they act in the same pathway cannot be excluded.

Interestingly no enhancement of $n f m-1$ PQR migration defects was seen in slt-1; nfm-1 double mutants. This indicates that slt-1 and $n f m-1$ interact in AQR migration but not PQR migration. This result, together with differential rescue of AQR $v s$. PQR defects by cell-specific $n f m-1$ (+) transgenes, suggests that NFM-1 might affect AQR and PQR differentially, possibly involving distinct expression levels, distinct sources of expression, or interactions with distinct genes.

sax-3/Robo mutants displayed both AQR and PQR migration defects. Possibly, SAX-3/Robo acts with SLT-1 in AQR migration, and with an unidentified ligand in PQR migration. In mice, midline axon defects are due to excess Slit2 
expression in NF2 mutants. The phenotypic enhancement that we observe between slt- 1 and $n f m-1$ suggests that these molecules are both required for AQR migration. Further studies of the interaction between $n f m-1$ and slt- 1 will be required to understand the role of these molecules in AQR migration. However, expression of $n f m-1$ from the slt- 1 promoter rescued AQR migration defects, suggesting that NFM-1 and SLT- 1 are required in the same tissues to control AQR migration, possibly the body wall muscles and/or the anal sphincter muscles where both are expressed.

Our results are consistent with the idea that NFM-1 promotes the production of a signal or signals that regulate $\mathrm{AQR}$ and $\mathrm{PQR}$ migration. This could be SLT-1 itself, such as in vertebrates, or a molecule that acts in parallel to SLT-1. Our combined results here suggest that NFM- 1 and SLT-1 might act in the body wall muscles and/or anal sphincter muscle. Of note, the Q-cell protrusions are in close proximity to body wall muscles (Figure 6), and the posterior body wall muscles are the source of SPON-1/ F-spondin, which is involved in Q migrations (Josephson et al. 2016b). Possibly, the posterior body wall muscles surrounding the $\mathrm{Q}$ neuroblasts serve as a source for cues that promote and guide their migrations. Further studies will be required to determine whether $n f m$ - 1 can control expression of guidance cue genes in muscles or if it is involved in secretion, adhesion, or extracellular matrix function to regulate a substrate for $\mathrm{Q}$ neuroblast migration.

\section{Acknowledgments}

The authors thank members of the Lundquist and Ackley labs for discussion, E. Struckhoff for technical assistance, and C. Bargmann for kyIs174. Some C. elegans strains were provided by the Caenorhabditis Genetics Center, which is funded by National Institutes of Health (NIH) Office of Research Infrastructure Programs (P40 OD010440). Some next-generation sequencing was provided by the University of Kansas Genome Sequencing Core Laboratory of the Center for Molecular Analysis of Disease Pathways (NIH P20 GM103638). This work was supported by NIH grants R01 NS040945 and R21 NS070417 to E.A.L. and the Kansas Infrastructure Network of Biomedical Research Excellence (NIH P20 GM103418). M.P.J. was supported by the Madison and Lila Self Graduate Fellowship at the University of Kansas, and R.A. and M.L.N. were Kansas IdeA Netword of Biomedical Research Excellence undergraduate research scholars (NIH P20 GM103418).

\section{Literature Cited}

Bagri, A., O. Marin, A. S. Plump, J. Mak, S. J. Pleasure et al., 2002 Slit proteins prevent midline crossing and determine the dorsoventral position of major axonal pathways in the mammalian forebrain. Neuron 33: 233-248.

Branda, C. S., and M. J. Stern, 2000 Mechanisms controlling sex myoblast migration in Caenorhabditis elegans hermaphrodites. Dev. Biol. 226: 137-151.
Chang, C., C. E. Adler, M. Krause, S. G. Clark, F. B. Gertler et al., 2006 MIG-10/lamellipodin and AGE-1/PI3K promote axon guidance and outgrowth in response to slit and netrin. Curr. Biol. 16: 854-862.

Chapman, J. O., H. Li, and E. A. Lundquist, 2008 The MIG-15 NIK kinase acts cell-autonomously in neuroblast polarization and migration in C. elegans. Dev. Biol. 324: 245-257.

Cordes, S., C. A. Frank, and G. Garriga, 2006 The C. elegans MELK ortholog PIG-1 regulates cell size asymmetry and daughter cell fate in asymmetric neuroblast divisions. Development 133: $2747-2756$.

Fehon, R. G., T. Oren, D. R. LaJeunesse, T. E. Melby, and B. M. McCartney, 1997 Isolation of mutations in the Drosophila homologues of the human Neurofibromatosis 2 and yeast CDC42 genes using a simple and efficient reverse-genetic method. Genetics 146: 245-252.

Gusella, J. F., V. Ramesh, M. MacCollin, and L. B. Jacoby, 1996 Neurofibromatosis 2: loss of merlin's protective spell. Curr. Opin. Genet. Dev. 6: 87-92.

Gutmann, D. H., M. J. Giordano, A. S. Fishback, and A. Guha, 1997 Loss of merlin expression in sporadic meningiomas, ependymomas and schwannomas. Neurology 49: 267-270.

Hamaratoglu, F., M. Willecke, M. Kango-Singh, R. Nolo, E. Hyun et al., 2006 The tumour-suppressor genes NF2/Merlin and expanded act through Hippo signalling to regulate cell proliferation and apoptosis. Nat. Cell Biol. 8: 27-36.

Hao, J. C., T. W. Yu, K. Fujisawa, J. G. Culotti, K. Gengyo-Ando et al., 2001 C. elegans slit acts in midline, dorsal-ventral, and anterior-posterior guidance via the SAX-3/Robo receptor. Neuron 32: 25-38.

Harris, J., L. Honigberg, N. Robinson, and C. Kenyon, 1996 Neuronal cell migration in C. elegans: regulation of Hox gene expression and cell position. Development 122: 3117-3131.

Harterink, M., D. H. Kim, T. C. Middelkoop, T. D. Doan, A. van Oudenaarden et al., 2011 Neuroblast migration along the anteroposterior axis of C. elegans is controlled by opposing gradients of Wnts and a secreted Frizzled-related protein. Development 138: 2915-2924.

Hilman, D., and U. Gat, 2011 The evolutionary history of YAP and the hippo/YAP pathway. Mol. Biol. Evol. 28: 2403-2417.

Honigberg, L., and C. Kenyon, 2000 Establishment of left/right asymmetry in neuroblast migration by UNC-40/DCC, UNC-73/ Trio and DPY-19 proteins in C. elegans. Development 127: 4655-4668.

Hunter, C. P., J. M. Harris, J. N. Maloof, and C. Kenyon, 1999 Hox gene expression in a single Caenorhabditis elegans cell is regulated by a caudal homolog and intercellular signals that inhibit wnt signaling. Development 126: 805-814.

Iwasa, H., S. Maimaiti, H. Kuroyanagi, S. Kawano, K. Inami et al., 2013 Yes-associated protein homolog, YAP-1, is involved in the thermotolerance and aging in the nematode Caenorhabditis elegans. Exp. Cell Res. 319: 931-945.

James, M. F., S. Han, C. Polizzano, S. R. Plotkin, B. D. Manning et al., $2009 \mathrm{NF} 2 /$ merlin is a novel negative regulator of mTOR complex 1 , and activation of mTORC1 is associated with meningioma and schwannoma growth. Mol. Cell. Biol. 29: 4250-4261.

Josephson, M. P., Y. Chai, G. Ou, and E. A. Lundquist, 2016a EGL-20/Wnt and MAB-5/Hox act sequentially to inhibit anterior migration of neuroblasts in C. elegans. PLoS One 11: e0148658.

Josephson, M. P., A. M. Miltner, and E. A. Lundquist, 2016b Nonautonomous roles of MAB-5/Hox and the secreted basement membrane molecule SPON-1/F-Spondin in Caenorhabditis elegans neuronal migration. Genetics 203: 1747-1762.

Kim, M., W. T. Farmer, B. Bjorke, S. A. McMahon, P. J. Fabre et al., 2014 Pioneer midbrain longitudinal axons navigate using a balance of Netrin attraction and Slit repulsion. Neural Dev. 9: 17. 
Korswagen, H. C., 2002 Canonical and non-canonical Wnt signaling pathways in Caenorhabditis elegans: variations on a common signaling theme. BioEssays 24: 801-810.

Lavado, A., M. Ware, J. Pare, and X. Cao, 2014 The tumor suppressor Nf2 regulates corpus callosum development by inhibiting the transcriptional coactivator Yap. Development 141: 4182-4193.

Maloof, J. N., J. Whangbo, J. M. Harris, G. D. Jongeward, and C. Kenyon, 1999 A Wnt signaling pathway controls hox gene expression and neuroblast migration in C. elegans. Development 126: 37-49.

McClatchey, A. I., I. Saotome, V. Ramesh, J. F. Gusella, and T. Jacks, 1997 The Nf2 tumor suppressor gene product is essential for extraembryonic development immediately prior to gastrulation. Genes Dev. 11: 1253-1265.

Mello, C., and A. Fire, 1995 DNA transformation. Methods Cell Biol. 48: 451-482.

Middelkoop, T. C., and H. C. Korswagen, 2014 Development and migration of the C. elegans $\mathrm{Q}$ neuroblasts and their descendants. WormBook 1-23.

Minevich, G., D. S. Park, D. Blankenberg, R. J. Poole, and O. Hobert, 2012 CloudMap: a cloud-based pipeline for analysis of mutant genome sequences. Genetics 192: 1249-1269.

Moroishi, T., H. W. Park, B. Qin, Q. Chen, Z. Meng et al., 2015 A YAP/TAZ-induced feedback mechanism regulates Hippo pathway homeostasis. Genes Dev. 29: 1271-1284.

Nguyen Ba-Charvet, K. T., K. Brose, V. Marillat, T. Kidd, C. S. Goodman et al., 1999 Slit2-Mediated chemorepulsion and collapse of developing forebrain axons. Neuron 22: 463-473.

Okada, M., Y. Wang, S. W. Jang, X. Tang, L. M. Neri et al., 2009 Akt phosphorylation of merlin enhances its binding to phosphatidylinositols and inhibits the tumor-suppressive activities of merlin. Cancer Res. 69: 4043-4051.

Okkema, P. G., S. W. Harrison, V. Plunger, A. Aryana, and A. Fire, 1993 Sequence requirements for myosin gene expression and regulation in Caenorhabditis elegans. Genetics 135: 385-404.

Pan, C. L., J. E. Howell, S. G. Clark, M. Hilliard, S. Cordes et al., 2006 Multiple Wnts and frizzled receptors regulate anteriorly directed cell and growth cone migrations in Caenorhabditis elegans. Dev. Cell 10: 367-377.

Piper, M., K. Georgas, T. Yamada, and M. Little, 2000 Expression of the vertebrate Slit gene family and their putative receptors, the Robo genes, in the developing murine kidney. Mech. Dev. 94: 213-217.

Quinn, C. C., D. S. Pfeil, E. Chen, E. L. Stovall, M. V. Harden et al., 2006 UNC-6/netrin and SLT-1/slit guidance cues orient axon outgrowth mediated by MIG-10/RIAM/lamellipodin. Curr. Biol. 16: 845-853.

Salser, S. J., and C. Kenyon, 1992 Activation of a C. elegans Antennapedia homologue in migrating cells controls their direction of migration. Nature 355: 255-258.

Salser, S. J., and C. Kenyon, 1996 A C. elegans Hox gene switches on, off, on and off again to regulate proliferation, differentiation and morphogenesis. Development 122: 1651-1661.

Salser, S. J., C. M. Loer, and C. Kenyon, 1993 Multiple HOM-C gene interactions specify cell fates in the nematode central nervous system. Genes Dev. 7: 1714-1724.

Sarov, M., S. Schneider, A. Pozniakovski, A. Roguev, S. Ernst et al., 2006 A recombineering pipeline for functional genomics applied to Caenorhabditis elegans. Nat. Methods 3: 839-844.

Schulz, A., A. Zoch, and H. Morrison, 2014 A neuronal function of the tumor suppressor protein merlin. Acta Neuropathol. Commun. 2: 82.
Schulz, A., S. L. Baader, M. Niwa-Kawakita, M. J. Jung, R. Bauer et al., 2013 Merlin isoform 2 in neurofibromatosis type 2-associated polyneuropathy. Nat. Neurosci. 16: 426-433.

Shakir, M. A., J. S. Gill, and E. A. Lundquist, 2006 Interactions of UNC-34 enabled with Rac GTPases and the NIK kinase MIG-15 in Caenorhabditis elegans axon pathfinding and neuronal migration. Genetics 172: 893-913.

Skop, A. R., H. Liu, J. Yates, III, B. J. Meyer, and R. Heald, 2004 Dissection of the mammalian midbody proteome reveals conserved cytokinesis mechanisms. Science 305: 61-66.

Steimel, A., J. Suh, A. Hussainkhel, S. Deheshi, J. M. Grants et al., 2013 The C. elegans CDK8 Mediator module regulates axon guidance decisions in the ventral nerve cord and during dorsal axon navigation. Dev. Biol. 377: 385-398.

Striedinger, K., S. R. VandenBerg, G. S. Baia, M. W. McDermott, D. H. Gutmann et al., 2008 The neurofibromatosis 2 tumor suppressor gene product, merlin, regulates human meningioma cell growth by signaling through YAP. Neoplasia 10: 1204-1212.

Sulston, J., and J. Hodgkin, 1988 Methods, pp. 587-606 in The Nematode Caenorhabditis elegans, edited by W. B. Wood, Cold Spring Harbor Laboratory Press, Cold Spring Harbor, NY.

Sulston, J. E., and S. Brenner, 1974 The DNA of Caenorhabditis elegans. Genetics 77: 95-104.

Sulston, J. E., and H. R. Horvitz, 1977 Post-embryonic cell lineages of the nematode, Caenorhabditis elegans. Dev. Biol. 56: 110-156.

Sundararajan, L., and E. A. Lundquist, 2012 Transmembrane proteins UNC-40/DCC, PTP-3/LAR, and MIG-21 control anteriorposterior neuroblast migration with left-right functional asymmetry in Caenorhabditis elegans. Genetics 192: 1373-1388.

Sundararajan, L., M. L. Norris, and E. A. Lundquist, 2015 SDN-1/ Syndecan acts in parallel to the transmembrane molecule MIG13 to promote anterior neuroblast migration. G3 5: 1537-1574.

Sundararajan, L., M. L. Norris, S. Schoneich, B. D. Ackley, and E. A. Lundquist, 2014 The fat-like cadherin CDH-4 acts cellnon-autonomously in anterior-posterior neuroblast migration. Dev. Biol. 392: 141-152.

Unni, D. K., M. Piper, R. X. Moldrich, I. Gobius, S. Liu et al., 2012 Multiple Slits regulate the development of midline glial populations and the corpus callosum. Dev. Biol. 365: 36-49.

Wang, X., F. Zhou, S. Lv, P. Yi, Z. Zhu et al., 2013 Transmembrane protein MIG-13 links the Wnt signaling and Hox genes to the cell polarity in neuronal migration. Proc. Natl. Acad. Sci. USA 110: $11175-11180$.

Whangbo, J., and C. Kenyon, 1999 A Wnt signaling system that specifies two patterns of cell migration in C. elegans. Mol. Cell 4: 851-858.

Xu, Y., and C. C. Quinn, 2012 MIG-10 functions with ABI-1 to mediate the UNC-6 and SLT-1 axon guidance signaling pathways. PLoS Genet. 8: e1003054.

Zhao, B., X. Wei, W. Li, R. S. Udan, Q. Yang et al., 2007 Inactivation of YAP oncoprotein by the Hippo pathway is involved in cell contact inhibition and tissue growth control. Genes Dev. 21: 2747-2761.

Zinovyeva, A. Y., and W. C. Forrester, 2005 The C. elegans Frizzled CFZ-2 is required for cell migration and interacts with multiple Wnt signaling pathways. Dev. Biol. 285: 447-461.

Zinovyeva, A. Y., Y. Yamamoto, H. Sawa, and W. C. Forrester, 2008 Complex network of Wnt signaling regulates neuronal migrations during Caenorhabditis elegans. Dev. Genet. 179: 1357-1371. 